\title{
A dual approach to structure constants for $K$-theory of Grassmannians
}

\author{
Huilan Li $\rrbracket$, Jennifer Morse非, and Pat Shields非 \\ ${ }^{1}$ School of Mathematical Sciences, Shandong Normal University, Jinan 250014, Shandong, China \\ ${ }^{2}$ Department of Mathematics, Drexel University, 15 S. 33rd Street, Philadelphia PA 19104, United States
}

\begin{abstract}
The problem of computing products of Schubert classes in the cohomology ring can be formulated as the problem of expanding skew Schur polynomial into the basis of ordinary Schur polynomials. We reformulate the problem of computing the structure constants of the Grothendieck ring of a Grassmannian variety with respect to its basis of Schubert structure sheaves in a similar way; we address the problem of expanding the generating functions for skew reverse-plane partitions into the basis of polynomials which are Hall-dual to stable Grothendieck polynomials. From this point of view, we produce a chain of bijections leading to Buch's $K$-theoretic Littlewood-Richardson rule.

Résumé. Le calcul des produits de classes de Schubert dans l'anneau de cohomologie correspond dans le langage des fonctions symétriques au développement des polynômes de Schur gauches dans la base des polynômes de Schur. Nous reformulons de façon similaire le calcul des constantes de structure de l'anneau de Grothendieck d'une variété grassmannienne dans la base des faisceaux de structure de Schubert; nous étudions le problème du développement des fonctions génératrices des partitions planes inversée gauches dans une base de polynômes duaux aux polynômes de Grothendieck stables. De cette reformulation s'ensuit une séquence de bijections qui permet de redériver la règle de Littlewood-Richardson en $K$-théorie obtenue par Buch.
\end{abstract}

Keywords. Grothendieck polynomial, Littlewood-Richardson rule, tabloid, sign-reversing involution, Yamanouchi word

\section{Introduction}

The theory of symmetric functions supports Schubert calculus of the Grassmannian $X=\operatorname{Gr}(k, n)$ by way of the Schur function basis. In particular, the Schubert cell decomposition of $X$ is given by the closures of Schubert varieties $X_{\lambda}$, for partitions $\lambda$ in a $k \times(n-k)$ rectangle. The cohomology classes corresponding to Schubert varieties form an integral basis for the cohomology ring of $X$, which in turn is isomorphic to a certain quotient of the ring of symmetric polynomials in $k$ variables. The Schur polynomials $s_{\lambda}\left(x_{1}, \ldots, x_{k}\right)$ are representatives for the Schubert classes with a critical feature that structure constants in

$$
\left[X_{\lambda}\right] \cdot\left[X_{\mu}\right]=\sum_{\nu} C_{\lambda \mu}^{\nu}\left[X_{\nu}\right]
$$

\footnotetext{
†Email: huilan.li@gmail.com.

¥Email: morse jemath. drexel. edu. She is supported by NSF grant DMS-1301695

§Email: prs49@drexel . edu. He is supported by NSF grant DMS-1301695
} 
appear as Schur coefficients in a product of Schur polynomials:

$$
s_{\lambda} s_{\mu}=\sum_{\nu} C_{\lambda \mu}^{\nu} s_{\nu}
$$

The realization of Schur polynomials as weight generating functions of semi-standard Young tableaux then offers combinatorial tools for the study. The development of a rich theory of tableaux ultimately enabled the computation of the structure constants $C_{\lambda \mu}^{\nu}$. The Littlewood-Richardson rule for $C_{\lambda \mu}^{\nu}$ dictates a count of tableaux with certain restrictions, and its proof was settled in Schützenberger (1977) using the RSK insertion algorithm on tableaux given by Robinson (1938); Schensted (1961); Knuth (1970).

Poincare duality allows for an alternative way to access the Schubert structure constants. Namely, the Poincaré dual of the product $\left[X_{\lambda}\right] \cdot\left[X_{\mu}\right]$ in $H^{*}(X)$ is identified by the skew Schur function $s_{\mu^{\vee} / \lambda}$, and the Littlewood-Richardson numbers arise in its ordinary Schur expansion:

$$
s_{\nu / \lambda}=\sum_{\mu} C_{\lambda \mu}^{\nu} s_{\mu}
$$

This viewpoint has since given rise to many simple proofs of the Littlewood-Richardson rule (e.g. Remmel and Shimozono (1998); Stembridge (2002)).

Developments in Schubert calculus have established the importance of combinatorics and symmetric function theory to the more intricate setting of the Grothendieck ring $K^{\circ} X$ of algebraic vector bundles on $X$. Lascoux and Schützenberger (1983) introduced Grothendieck polynomials as representatives for the structure sheaves of the Schubert varieties in a flag variety. Fomin and Kirillov (1994) studied the symmetric power series resulting from a limit of Grothendieck polynomials. For the Grassmannian variety $X$, Buch (2002) showed that the stable limits $G_{\lambda}$ are generating series of set-valued tableaux and can be applied to $K^{\circ} X$ in a way that mirrors the Schur role in cohomology.

The classes of the Schubert structure sheaves $\mathcal{O}_{X_{\lambda}}$ form a basis for the Grothendieck ring of $X$ and the structure constants in

$$
\left[\mathcal{O}_{X_{\lambda}}\right] \cdot\left[\mathcal{O}_{X_{\mu}}\right]=\sum_{\nu} c_{\lambda \mu}^{\nu}\left[\mathcal{O}_{X_{\nu}}\right]
$$

appear in the product

$$
G_{\lambda} G_{\mu}=\sum_{\nu} c_{\lambda \mu}^{\nu} G_{\nu}
$$

By generalizing the RSK insertion, Buch (2002) proved that the expansion coefficients are $(-1)^{|\nu|-|\lambda|-|\mu|}$ times the number of set-valued tableaux with a Yamanouchi reading word.

In contrast to (1), the Schubert structure constant $c_{\lambda \mu}^{\nu}$ for $K^{\circ} X$ is not the coefficient of $G_{\mu}$ in $G_{\nu / \lambda}$. However, we have found a dual approach that does give access to the constants using a set of representatives $\left\{g_{\lambda}\right\}$ for classes in $K$-homology of the ideal sheaves of the boundaries of $X_{\lambda}$. The combinatorial investigations of Lam and Pylyavskyy (2007) revealed that these representatives can be realized as the weight generating function of reverse plane partitions. Here we reach the $K$-theoretic structure constants through the more general class of generating functions of reverse plane partitions on skew shapes, $g_{\nu / \lambda}$. In particular,

$$
g_{\nu / \lambda}=\sum_{\mu} c_{\lambda \mu}^{\nu} g_{\mu}
$$


We convert the weight generating function characterization for $g_{\nu / \lambda}$ into a setting that instead uses tabloid, a classical combinatorial object going back to Young and the study of $S_{n}$-irreducibles. The association between reverse plane partitions and tabloids requires an unconventional notion of weight called the dilated weight. We prove a number of properties such as a dilated weight formulation for the Yamanouchi condition. From this, a series of natural bijections leads to Buch's $K$-theoretic LittlewoodRichardson rule for $c_{\lambda \mu}^{\nu}$.

\section{Preliminaries}

Let $X=\operatorname{Gr}(k, n)$ denote the Grassmannian of $k$-dimensional subspaces of $\mathbb{C}^{n} . X$ decomposes into Schubert cells, whose closures are the Schubert varieties

$$
X_{\lambda}=\left\{V \in \operatorname{Gr}(k, n): \operatorname{dim}\left(V \cap \mathbb{C}^{n-k+i-\lambda_{i}}\right) \geq i \forall 1 \leq i \leq k\right\},
$$

for partitions $\lambda=\left(\lambda_{1}, \ldots, \lambda_{k}\right)$ with $k$ parts, none of which are larger than $n-k$. Here $\mathbb{C}^{d}$ denotes the subspace of $\mathbb{C}^{n}$ where vectors have only non-zero entries in the first $d$ components. The classes of Schubert cells form a basis for the singular cohomology of $X$.

On the other hand, $H^{*}(X)$ is isomorphic to a certain quotient of the ring of symmetric polynomials. The ring of symmetric functions is $\Lambda=\mathbb{Z}\left[e_{1}, \ldots, e_{k}\right]=\mathbb{Z}\left[h_{1}, \ldots, h_{k}\right]$, where

$$
e_{r}=\sum_{1 \leq i_{1}<\cdots<i_{r}} x_{i_{1}} \cdots x_{i_{r}} \quad \text { and } \quad h_{r}=\sum_{1 \leq i_{1} \leq \cdots \leq i_{r}} x_{i_{1}} \cdots x_{i_{r}}
$$

As a vector space, the bases for $\Lambda$ are indexed by generic non-decreasing integer partitions $\lambda=\left(\lambda_{1}, \lambda_{2}, \ldots, \lambda_{\ell}\right)$. A quick example is the basis of homogenous symmetric functions, defined by $h_{\lambda}=h_{\lambda_{1}} \cdots h_{\lambda_{\ell}}$.

The combinatorial potential of $\Lambda$ could not be met without the unique association of each partition $\lambda$ with its Ferrers shape, a left-and bottom-justified array of $1 \times 1$ square cells in the first quadrant of the coordinate plane, with $\lambda_{i}$ cells in the $i^{\text {th }}$ row from the bottom. Given a partition $\lambda$, its conjugate $\lambda^{\prime}$ is the partition obtained by reflecting the shape of $\lambda$ about the line $y=x$. For partitions $\mu, \lambda, \mu \subset \lambda$ when every cell of $\mu$ is a cell of $\lambda$. For $\mu \subset \lambda$, the skew shape $\lambda / \mu$ is defined by the cells in $\lambda$ but not in $\mu$. For any partitions $\mu$ and $\lambda, \mu * \lambda$ is the skew shape obtained by placing the diagram of $\lambda$ southeast and caty-corner to $\mu$. For example,

$$
\mu=(2,2,1), \lambda=(3,1) \Longrightarrow \mu * \lambda=(5,3,2,2,1) /(2,2)=\frac{\square}{\square}
$$

More generally, a composition is a sequence of non-negative integers $\alpha=\left(\alpha_{1}, \alpha_{2}, \ldots, \alpha_{k}\right)$, and $\alpha-\beta$ is defined by usual vector subtraction for any compositions $\alpha, \beta$.

A semi-standard tableau of shape $\lambda$ is a positive integer filling of the cells of $\lambda$ such that entries do not decrease from left to right in rows and are increasing from the bottom to top of each column. The weight of a tableau $T$ denoted wt(T) is the composition $\alpha=\left(\alpha_{i}\right)_{i \geq 1}$ where $\alpha_{i}$ is the number of cells containing $i$ (it is customary to omit trailing 0 's). We use $\operatorname{SSYT}(\lambda)$ to denote the set of all semi-standard tableaux of shape $\lambda$, and $\operatorname{SSYT}(\lambda, \mu)$ to denote the set of all semi-standard tableaux of shape $\lambda$ and weight $\mu$. 
The weight generating functions of semi-standard tableaux define the Schur basis; for any partition $\lambda$,

$$
s_{\lambda}=\sum_{T \in \operatorname{SSYT}(\lambda)} \mathbf{x}^{\mathrm{wt}(\mathrm{T})}
$$

where $\mathbf{x}^{\alpha}=x_{1}^{\alpha_{1}} x_{2}^{\alpha_{2}} \cdots$. It is convenient to collected terms into the basis of monomials, formed by $m_{\lambda}=\sum_{\alpha} \mathbf{x}^{\alpha}$, for all rearrangements $\alpha$ of partition $\lambda$. The monomial expansion of Schur functions is

$$
s_{\lambda}=\sum_{\mu} K_{\lambda, \mu} m_{\mu}
$$

where the Kostka numbers $K_{\lambda, \mu}$ enumerate semi-standard tableaux of shape $\lambda$ and weight $\mu$, for partitions $\lambda, \mu$.

The Schur basis is orthonormal with respect to the Hall-inner product $\langle$,$\rangle on \Lambda$, defined by

$$
\left\langle m_{\lambda}, h_{\mu}\right\rangle=\left\{\begin{array}{lc}
1 & \text { if } \lambda=\mu \\
0 & \text { otherwise }
\end{array}\right.
$$

An immediate consequence of (3) and duality is that

$$
h_{\mu}=\sum_{\lambda} K_{\lambda, \mu} s_{\lambda}
$$

Our methods rely on the fundamental operations on words and tableaux such as jeu de taquin due to Schützenberger (1977) and RSK-insertion due to Robinson (1938); Schensted (1961); Knuth (1970). We briefly recall several important results here and full details can be found in a variety of texts such as Lascoux and Schützenberger (1981); Stanley (1999); Fulton (1997). The reading word $\mathrm{w}(\mathrm{T})=\mathrm{w}_{1} \mathrm{w}_{2} \cdots \mathrm{w}_{\mathrm{n}}$ of any filling $T$ is defined by listing elements of $T$ starting from the top-left corner, reading across each row, and then continuing down the rows. A word $w$ is Yamanouchi when each factorization $w=u v$ satisfies $\mathrm{wt}_{i}(v) \geq \mathrm{wt}_{i+1}(v)$ where $\mathrm{wt}_{i}(v)$ is the number of times letter $i$ appears in $v$. For a given partition $\lambda$, a word $w$ is $\lambda$-Yamanouchi if $\mathrm{wt}_{i}(v)+\lambda_{i} \geq \mathrm{wt}_{i+1}(v)+\lambda_{i+1}$. The RSK bijection identifies a word $w$ with a pair of same shaped tableaux $(P(w), Q(w))$. For any tableau $T$,

$$
P(\mathrm{w}(\mathrm{T}))=\mathrm{T},
$$

and when two words $w$ and $u$ have the same insertion tableau $P(w)=P(u)$, they are Knuth equivalent, denoted by $w \sim u$.

\section{$3 \quad K$-theoretic Littlewood-Richardson coefficients}

Because the open Schubert cells form a cell decomposition of the Grassmannian $X$, the classes of the structure sheaves $\mathcal{O}_{X_{\lambda}}$ form a basis for the Grothendieck ring of $X$. The structure constants of $K^{\circ} X$ with respect to its basis of Schubert structure sheaves, appearing in

$$
\left[\mathcal{O}_{X_{\lambda}}\right] \cdot\left[\mathcal{O}_{X_{\mu}}\right]=\sum_{\nu} c_{\lambda \mu}^{\nu}\left[\mathcal{O}_{X_{\nu}}\right]
$$

was solved in Buch (2002). Buch's work initiated a tableaux combinatorial framework to study $K$ theoretic Schubert calculus. 


\subsection{Combinatorics of $K$-theory classes}

A set-valued tableau of shape $\nu / \lambda$ is a filling of each cell in the diagram of $\nu / \lambda$ with a non-empty set of positive integers such that each subfilling created from the choice of a single element in each cell is a semi-standard tableau. The weight of a set-valued tableau $S$ is the composition $\alpha=\left(\alpha_{i}\right)_{i \geq 1}$ where $\alpha_{i}$ is the total number of times $i$ appears in $S$ and the excess of $S$ is defined by

$$
\varepsilon(S)=|\operatorname{wt}(\mathrm{S})|-|\operatorname{shape}(\mathrm{S})| .
$$

A multicell refers to a cell in $S$ that contains more than one letter. When $S$ has no multicells, it is viewed as a semi-standard tableau. In this case, $|\operatorname{wt}(\mathrm{S})|=|\operatorname{shape}(\mathrm{S})|$ and $\varepsilon(S)=0$.

The collection of all set-valued tableaux of shape $\nu / \lambda$ is denoted by $\operatorname{SVT}(\nu / \lambda)$ and the subset of these with weight $\alpha$ is $\operatorname{SVT}(\nu / \lambda, \alpha)$. For any partitions $\nu \supset \lambda$ and composition $\alpha$, let

$$
k_{\nu / \lambda, \alpha}=\sum_{S \in \operatorname{SVT}(\nu / \lambda, \alpha)}(-1)^{\varepsilon(S)} .
$$

Theorem 1 (Buch (2002)) For any partition $\lambda$,

$$
G_{\lambda}=\sum_{S \in \operatorname{SVT}(\lambda)}(-1)^{\varepsilon(S)} x^{\mathrm{wt}(\mathrm{S})}=\sum_{\mu} k_{\lambda \mu} m_{\mu} .
$$

The span of stable Grothendieck polynomials is a bi-algebra $\Gamma$ and the Grothendieck ring $K^{\circ} X$ is isomorphic to $\Gamma$ modulo the ideal spanned by the subset of $G_{\lambda}$ with $\lambda$ not fitting inside a $k \times(n-k)$ rectangle. The completion $\mathfrak{m S y m}$ of $\Gamma$ is a Hopf algebra, and its dual $\mathfrak{M S y m}$ of multisymmetric functions is equipped with a distinguished basis $\left\{g_{\lambda}\right\}$ that is dual to $\left\{G_{\lambda}\right\}$. That is, $g_{\lambda}$ is defined by inverting the triangular system

$$
h_{\mu}=\sum_{\lambda} k_{\lambda, \mu} g_{\lambda}
$$

over all partitions $\mu$. This basis is a set of representatives for classes in $K$-homology of the ideal sheaves of the boundaries of Schubert varieties.

Alternatively, $g_{\lambda}$ can be combinatorial characterized as certain weight generating functions of reverse plane partitions of partition shape $\lambda$. Define a reverse plane partition $R$ of shape $\nu / \lambda$ to be a filling of cells in $\nu / \lambda$ with positive integers which are weakly increasing in rows and columns. The weight of $R$ is the composition $\alpha=\left(\alpha_{i}\right)_{i \geq 1}$ where $\alpha_{i}$ is the total number of columns of $R$ in which $i$ appears. The collection of all reverse plane partitions of shape $\nu / \lambda$ will be denoted $\operatorname{RPP}(\nu / \lambda)$ and the subset of these with weight $\alpha$ will be $\operatorname{RPP}(\nu / \lambda, \alpha)$.

Theorem 2 (Lam and Pylyavskyy (2007)) The polynomials $g_{\lambda}$ have the expansion

$$
g_{\lambda}=\sum_{R \in \operatorname{RPP}(\lambda)} \mathbf{x}^{\mathrm{wt}(\mathrm{R})} .
$$


Lenart (2000) had shown previously that the transition matrices between Schur functions and the functions $G_{\mu}$ can be described using strict elegant fillings. These are skew semi-standard tableaux which are strictly increasing in both rows and columns, and with the property that numbers in row $i$ are not larger than $i-1$. The set of all strict elegant fillings of shape $\lambda / \mu$ is denoted by $\operatorname{EF}(\lambda / \mu)$ and we set $F_{\lambda}^{\mu}=|\operatorname{EF}(\lambda / \mu)|$.

Theorem 3 (Lenart (2000)) For any partition $\lambda$,

$$
G_{\mu}=\sum_{\lambda}(-1)^{|\lambda|-|\mu|} F_{\mu}^{\lambda} s_{\lambda} .
$$

An immediate corollary comes out of the duality relation:

$$
s_{\lambda}=\sum_{\mu}(-1)^{|\lambda|-|\mu|} F_{\mu}^{\lambda} g_{\mu} .
$$

\section{2 $K$-theoretic structure constants}

Buch (2002) discovered that the structure constants for Grothendieck functions can be described by defining a reading word on set-valued tableaux and selecting only those which are Yamanouchi. The (column) reading word $\mathrm{w}(\mathrm{S})$ of a set-valued tableau $S$ is defined by reading top to bottom across columns from left to right where letters within a cell are read from smallest to largest.

Example 4

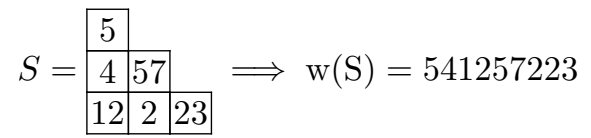

If $\mathrm{w}(\mathrm{S})$ is $\lambda$-Yamanouchi, we say $S$ is a $\lambda$-Yamanouchi set-valued tableau. We sometimes say a setvalued tableau is column Yamanouchi to emphasize that the property is determined by the column reading word.

Theorem 5 (Buch (2002)) For partitions $\lambda, \mu$,

$$
G_{\lambda} G_{\mu}=\sum_{|\nu| \geq|\lambda|+|\mu|} c_{\lambda \mu}^{\nu} G_{\nu}
$$

where $c_{\lambda \mu}^{\nu}$ is equal to $(-1)^{|\nu|-|\lambda|-|\mu|}$ times the number of $\lambda$-Yamanouchi set-valued tableaux of shape $\mu$ and weight $\nu-\lambda$.

We instead access the coefficients by appealing to the $g$-expansion of skew dual functions,

$$
g_{\nu / \lambda}=\sum_{\mu} r_{\nu / \lambda, \mu} m_{\mu}
$$

where $r_{\nu / \lambda, \mu}=|\operatorname{RPP}(\nu / \lambda, \mu)|$.

Lemma 6 For partitions $\mu \subseteq \nu$,

$$
g_{\nu / \mu}=\sum_{\lambda} c_{\mu \lambda}^{\nu} g_{\lambda}
$$




\section{Dilated weight}

A by-product of Theorem 5 and Lemma 6 is that the coefficient of $g_{\mu}$ in $g_{\nu / \lambda}$ is determined by counting skew Yamanouchi set-valued tableaux. Our interest is in giving a direct combinatorial proof for the $K$ theoretic Littlewood-Richardson rule starting from the skew reverse plane partition expansion for $g_{\nu / \lambda}$.

We largely avoid working with reverse plane partitions by identifying them with tabloids, fillings with positive integers that are not decreasing in rows. Tabloids are classical combinatorial objects, going back to Young's definition of the irreducible representations of $S_{n}$. However, our purposes require an association of tabloids (and set-valued tableaux) with a less familiar weight called the dilated weight. We start with the definition and several results establishing that the notion of this particular weight is closely tied to the Yamanouchi property.

\subsection{Dilated weight characterization for Yamanouchi objects}

The dilated weight $\delta \mathrm{wt}(T)$ of a tabloid $T$ is defined iteratively from its rows $T_{1}, T_{2}, \ldots, T_{\ell}$, read from bottom to top. With $r=2$ and $\hat{T}=T_{1}$, modify $T_{r} * \hat{T}$ by moving the last letter $e$ in $T_{r}$ to the rightmost empty cell of row $r$ that has no entry $e^{\prime} \geq e$ below it in any row $r^{\prime} \leq r-1$. If no such cell exists, $e$ remains in place. Ignoring $e$, repeat with the last letter in row $r$. Once all letters in row $r$ have been addressed, iterate by setting the resulting filling to $\hat{T}$ and $r=r+1$. When $r=\ell$, the process terminates with a column-strict filling $\hat{T}$ called the dilated weight tableau of $T$. The dilated weight is the conjugate of the partition rearrangement of the list of (uncovered) entries at the top of each column in $\hat{T}$.

\section{Example 7}

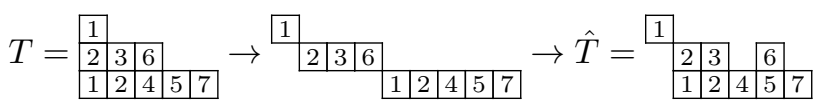

implying that $\delta \mathrm{wt}(T)=(7,6,4,3,2,1)^{\prime}=(6,5,4,3,2,2,1)$

A more general notion of skew dilated weight is defined on tabloid $T$. We say $\delta \mathrm{wt}(T)=\nu / \lambda$ when $\delta \operatorname{wt}\left(T * T_{\lambda}\right)=\nu$ for the unique tableau $T_{\lambda}$ of shape and weight $\lambda$. Our work involves the set $\mathcal{T}(\nu / \lambda)$ of tabloids of shape $\nu / \lambda$ and its restriction to the subset $\mathcal{T}(\nu / \lambda, \alpha)$ of tabloid with weight $\alpha$ and the subset $\mathcal{T}(\nu / \lambda, \delta \mathrm{wt}=\alpha)$ with dilated weight $\alpha$.

Example 8 The tabloid $T=\frac{1}{1}_{1_{2}}$ has skew dilated weight $\delta \mathrm{wt}(T)=(5,2) /(3,1)$ since

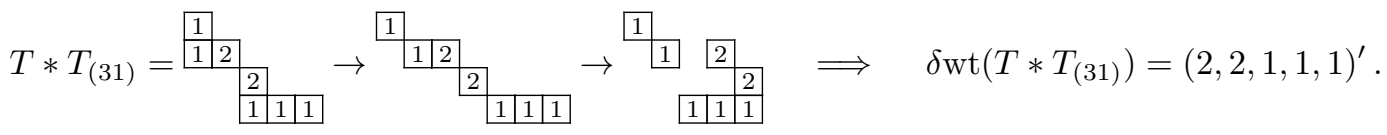

Note in this example that the weight of $T * T_{(31)}$ equals its dilated weight. In fact, this happens only when $T * T_{\lambda}$ is Yamanouchi - that is, it has a Yamanouchi reading word.

Proposition 9 For any tabloid $T$ and partition $\lambda$,

$$
T * T_{\lambda} \text { is Yamanouchi } \Longleftrightarrow \delta \mathrm{wt}\left(T * T_{\lambda}\right)=\operatorname{wt}\left(\mathrm{T} * \mathrm{~T}_{\lambda}\right) .
$$

Definition 10 The dilated weight of a set-valued tableau $S$ is defined iteratively from its rows $S_{1}, S_{2}, \ldots, S_{\ell}$, read from bottom to top. With $r=2$ and $\hat{S}=S_{1}$, starting from the last cell c in $S_{r}$, modify $S_{r} * \hat{S}$ as follows: 
1. move the largest entry e in cell $c$ to the rightmost empty cell $(r, j)$ such that all entries in cells $\left(r^{\prime}, j\right)$ for all $r^{\prime}<r$ are smaller than e. If no such cell exists, e remains in place.

2. move the next largest entry $e_{2}$ in cell c to the rightmost cell $(r, \hat{j})$, where $\hat{j} \leq j$, such that all entries in cells $\left(r^{\prime}, \hat{j}\right)$ for all $r^{\prime}<r$ are less than $e_{2}$. Let $j=\hat{j}$ and repeat this step on all remaining entries in cell $c$.

3. repeat from step 1 on the cell $\hat{c}$ just west of cell $c$.

When all cells in row $r$ have been addressed, iterate by setting the resulting filling to $\hat{S}$ and $r=r+1$. The process terminates when $r=\ell$, with a column-strict set-valued filling $\hat{S}$ called the dilated weight tableau of $S$. The dilated weight is the conjugate of the partition rearrangement of the list of maximal entries in uncovered cells of $\hat{S}$.

\section{Example 11}

$$
\begin{aligned}
& S=\begin{array}{|c|c|}
\hline 48 & 9 \\
\hline 1 & 5 \\
\hline
\end{array} \mapsto \begin{array}{c|c|}
\hline 48 & 9 \\
\hline 1 & 5 \\
\hline
\end{array} \quad \Longrightarrow \delta \mathrm{wt}(S)=(9,8)^{\prime}=(2,2,2,2,2,2,2,2,1)
\end{aligned}
$$

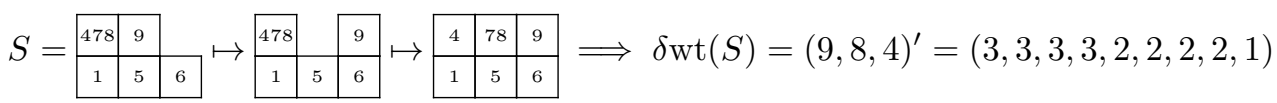

Remark 12 The dilated weight of a set-valued tableau could also have been defined as an iterative process on its columns. Reading the columns $S_{1}, S_{2}, \ldots, S_{\lambda_{1}}$ from left to right, first consider just the subdiagram with columns $S_{\lambda_{1}-1}$ and $S_{\lambda_{1}}$. Perform the moves described above starting with the lowest cell of $S_{\lambda_{1}-1}$ and working upwards. Repeat this process with the cells of $S_{\lambda_{1}-2}$ and proceed in this fashion until all columns are exhausted. Note that any cell $c=(r, j)$ in a set-valued tableau has entries which are strictly larger than the entries in cell $\left(r^{\prime}, j^{\prime}\right)$ for $r^{\prime} \leq r, j^{\prime} \leq j$. If we instead construct the dilated tableau by rows, all entries of c necessarily move further to the right than the entries in $\left(r^{\prime}, j^{\prime}\right)$. With this in mind one can see the resulting dilated weights to be equal.

Our result characterizing Yamanouchi tabloids by the property that dilated weight equals to weight extends to the set-valued framework as well.

Lemma 13 For partition $\lambda$ and set-valued tableau $S$, if $S * T_{\lambda}$ is (column) Yamanouchi then $\mathrm{wt}\left(\mathrm{S} * \mathrm{~T}_{\lambda}\right)=$ $\delta \mathrm{wt}\left(\mathrm{S} * \mathrm{~T}_{\lambda}\right)$ and the columns of the dilated weight tableau of $S * T_{\lambda}$ are interval valued.

\subsection{Reverse plane partitions and tabloids}

Dilated weight exposes a correspondence between tabloids and reverse plane partitions.

Proposition 14 (Kaliszewski and Morse) For any skew partition $\nu / \lambda$ and composition $\alpha$, there is a bijection

$$
\operatorname{RPP}(\nu / \lambda, \alpha) \longleftrightarrow \mathcal{T}(\alpha, \delta \mathrm{wt}=\nu / \lambda)
$$

The jeu de taquin operation induces a crystal graph on the set of tabloids with fixed dilated weight. The highest weights are simply tabloid with strictly increasing columns.

Lemma 15 (Kaliszewski and Morse) For each skew partition $\nu / \lambda$, a crystal graph supports $g_{\nu / \lambda}$ by taking vertices to be the set of tabloids $\mathcal{T}(\cdot, \delta \mathrm{wt}=\nu / \lambda)$. The highest weights are semi-standard tableaux of partition shape and dilated weight $\nu / \lambda$. 
A different perspective is studied by Galashin (2014) where a crystal graph on reverse plane partition is introduced. We use the tabloid point of view as it is amenable to a bijection $\phi$ of Bandlow and Morse (2012) associating set-valued tableaux to strict elegant fillings. This route gives us access to the $K$ theoretic Littlewood-Richardson structure constants.

The map $\phi$ on set-valued tableaux is defined by iteratively eliminating multicells through a process called dilation. Given a set-valued tableau $S$, let $\operatorname{row}(S)$ be the highest row containing a multicell. Let $S_{>i}$ denote the subtableau formed by taking only rows of $S$ above row $i$. For the rightmost multicell $c$ in row $(S)$, define $x=x(S)$ to be the largest entry in $c$. The dilation of $S, \operatorname{di}(S)$, is constructed from $S$ by removing $x$ from $c$ and RSK-inserting $x$ into $S_{>\operatorname{row}(S)}$.

Example 16 Since $\operatorname{row}(S)=2$ and $x(S)=6$,

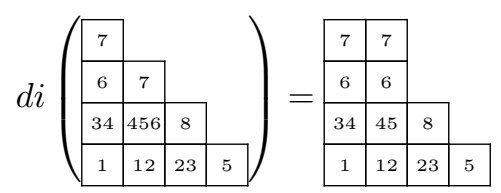

It was proven (Bandlow and Morse, 2012, Property 4.4) that dilation preserves Knuth equivalence. More precisely, for any set-valued tableau $S$,

$$
\operatorname{sw}(S) \sim \operatorname{sw}(\operatorname{di}(S))
$$

where the set-valued reading word $\operatorname{sw}(S)$ is the word obtained by listing the entries from rows of $S$ as follows (starting in the highest row): first ignore the smallest entry in each cell and record the remaining entries in the row from right to left and from largest to smallest within each cell, then record the smallest entry of each cell from left to right.

\section{Example 17}

$$
S=\begin{array}{|l|l|l|}
\hline 3 & 456 \\
\hline 12 & 23 & 3 \\
\hline
\end{array}
$$

Dilation expands a set-valued tableau by reducing the number of entries in a given multicell by one. The iteration of this process produces a semi-standard tableau from a set-valued tableau.

Definition 18 The map $\phi$ acts on a set valued tableau $S$ by constructing the sequence of set-valued tableaux

$$
S=S_{0} \rightarrow \operatorname{di}(S)=S_{1} \rightarrow \operatorname{di}\left(S_{1}\right)=S_{2} \rightarrow \cdots \rightarrow S_{r}
$$

and defining $\phi(S)$ to be the filling of $\operatorname{sh}\left(\mathrm{S}_{\mathrm{r}}\right) / \operatorname{sh}(\mathrm{S})$ where cell $\operatorname{sh}\left(\mathrm{S}_{\mathrm{i}}\right) / \operatorname{sh}\left(\mathrm{S}_{\mathrm{i}-1}\right)$ contains the difference between the row index of this cell and $\operatorname{row}\left(S_{i-1}\right)$. The sequence 10 is defined to terminate at the first set-valued tableau $S_{r}$ with no multicell.

\section{Example 19}

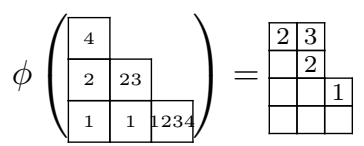


is constructed by recording the sequence of dilations

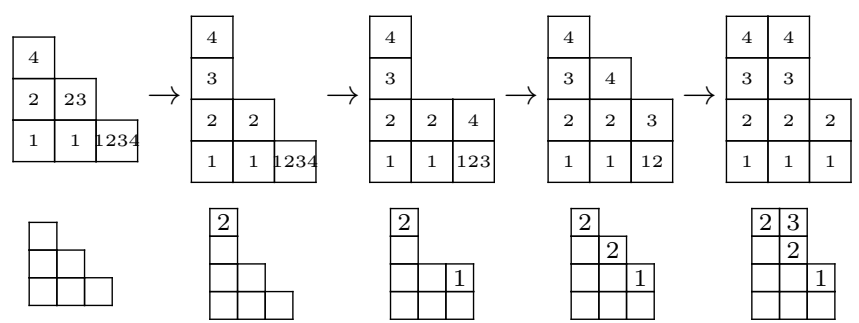

The restriction of $\phi$ to an action on a subset of set-valued tableaux determined by their set-valued reading words and a tableau $T$ is a bijection $\phi_{T}$ between these set-valued tableaux and elegant fillings. In particular this gives a bijective proof of Lenart's formula (Lenart, 2000, Theorem 3.2).

Proposition 20 (Bandlow and Morse (2012), Proposition 5.6) For any fixed tableau $T$ and partition $\eta \subset \operatorname{shape}(T)$,

$$
\phi_{T}:\{S \in \operatorname{SVT}(\eta): \operatorname{sw}(S) \sim \mathrm{w}(\mathrm{T})\} \leftrightarrow \operatorname{EF}(\operatorname{shape}(\mathrm{T}) / \eta)
$$

is a bijection.

Our purposes require a refinement of this result which identifies strict elegant fillings with subsets of set-valued tableaux characterized by dilated weight.

Proposition 21 For each $T \in \operatorname{SSYT}(\mu, \delta \mathrm{wt}=\alpha)$ and partition $\eta \subset \mu$,

$$
\phi_{T}:\{S \in \operatorname{SVT}(\eta, \delta \mathrm{wt}=\alpha): \operatorname{sw}(S) \sim \mathrm{w}(\mathrm{T})\} \leftrightarrow \mathrm{EF}(\operatorname{shape}(\mathrm{T}) / \eta)
$$

is a bijection.

\section{Dual approach to the $K$-theoretic LR rule}

Lemma 22 For partitions $\eta, \nu$, and $\lambda \subset \nu$, there is a sign-reversing involution on $\operatorname{SVT}(\eta, \delta \mathrm{wt}=\nu / \lambda)$ where the $\lambda$-Yamanouchi set-valued tableaux are fixed points.

We produce such a map $\tau$ which is shape preserving and is defined to fix set-valued tableaux which are $\lambda$-Yamanouchi. A similar involution was given independently by (Ikeda and Shimazaki, 2014, Lemma 3). Let $S_{\geq j}$ denoted the set-valued tableau obtained by considering only those cells which are weakly right of column $j$. When $S \in \operatorname{SVT}(\eta, \delta$ wt $=\nu / \lambda)$ is not $\lambda$-Yamanouchi, define $c$ to be the rightmost column such that $S_{\geq c}$ is not $\lambda$-Yamanouchi. Let $y$ be the rightmost letter in the reading word w $\left(\mathrm{S}_{\geq c}\right) \mathrm{w}\left(\mathrm{T}_{\lambda}\right)$ with the property that there are more $y$ 's than $y-1$ 's and take $r$ to be the row of the cell $(r, c)$ in $S$ containing this $y$. Denote by cell $l_{\min }$ the leftmost cell in row $r$ containing $y$. The image $\hat{S}=\tau(S)$ is defined by deleting $y-1$ if it is present in $c e l l_{\min }$ and otherwise $\hat{S}$ is obtained by adding $y-1$ to cell $_{\min }$.

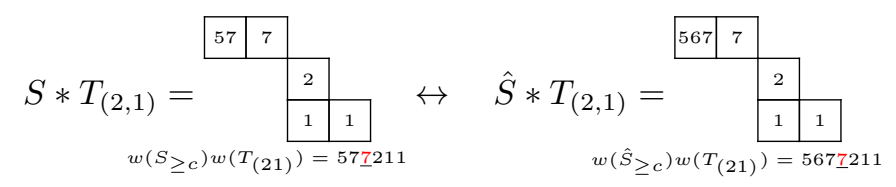

Theorem 23 For partitions $\mu$ and $\lambda \subset \nu$, the coefficient of $g_{\mu}$ in $g_{\nu / \lambda}$ is $(-1)^{|\nu|-|\lambda|-|\mu|}$ times the number of $\lambda$-Yamanouchi set-valued tableaux of shape $\mu$ and weight $\nu / \lambda$. 


\section{Related Work}

A recent breakthrough in modern Schubert calculus concerns the Grothendieck ring $K_{T}(X)$ of $T$-equivariant vector bundles over $X=\operatorname{Gr}(a, n)$. There is a natural $K_{T}(\mathrm{pt})$-module structure and an additive basis given by the classes of Schubert structure sheaves. The structure constants are now Laurent polynomials $c_{\lambda \mu}^{\nu}(t) \in \mathbb{Z}\left[t_{1}^{ \pm 1}, \ldots, t_{n}^{ \pm 1}\right] \simeq K_{T}(\mathrm{pt})$ appearing in

$$
\left[\mathcal{O}_{X_{\lambda}}\right] \cdot\left[\mathcal{O}_{X_{\mu}}\right]=\sum_{\nu \in k \times(n-k)} c_{\lambda \mu}^{\nu}(t)\left[\mathcal{O}_{X_{\nu}}\right]
$$

The family of $c_{\lambda \mu}^{\nu}(t)$ includes both the set of $K$-theoretic and equivariant structure constants on $X$. Groundbreaking work of Pechenik and Yong (2015) identified $c_{\lambda \mu}^{\nu}(t)$ by a new class of ballot genomic tableaux using a deep generalization of jeu de taquin. A special case of their work yields an alternate rule for the (non-equivariant) $K$-theory structure constants.

Additionally, Ikeda and Shimazaki (2014) provided a description of the structure constants for expanding the product $G_{\lambda}(\beta) G_{\mu / \eta}(\beta)$ in terms of the basis given by $G_{\nu}(\beta)$. The $G_{\nu}(\beta)$ are obtained by replacing the $(-1)$ in Theorem 1 by a parameter $\beta$. This provides a rule which when specialized reduces to that of Buch's for the $K$-theory structure constants.

\section{Acknowledgements}

The authors thank Ryan Kaliszewski for many discussions about related work.

\section{References}

J. Bandlow and J. Morse. Combinatorial expansions in K-theoretic bases. Electron. J. Combin., 19(4): Paper 39, 27, 2012. ISSN 1077-8926.

A. S. Buch. A Littlewood-Richardson rule for the K-theory of Grassmannians. Acta Math., 189(1):3778, 2002. ISSN 0001-5962. doi: 10.1007/BF02392644. URL http://dx.doi.org/10.1007/ BF 02392644 .

S. Fomin and A. N. Kirillov. Grothendieck polynomials and the Yang-Baxter equation. In Formal power series and algebraic combinatorics/Séries formelles et combinatoire algébrique, pages 183-189. DIMACS, Piscataway, NJ, 1994.

W. Fulton. Young tableaux, volume 35 of London Mathematical Society Student Texts. Cambridge University Press, Cambridge, 1997. ISBN 0-521-56144-2; 0-521-56724-6. With applications to representation theory and geometry.

P. Galashin. A Littlewood-Richardson Rule for Dual Stable Grothendieck Polynomials. ArXiv e-prints, Dec. 2014.

T. Ikeda and T. Shimazaki. A proof of k-theoretic Littlewood-Richardson rules by Bender-Knuth-type involutions. Math. Res. Lett, 21(2):333-339, 2014.

R. Kaliszewski and J. Morse. Airports for equivariant $k$-theory of grassmannians. 
D. E. Knuth. Permutations, matrices, and generalized Young tableaux. Pacific J. Math., 34:709-727, 1970. ISSN 0030-8730.

T. Lam and P. Pylyavskyy. Combinatorial Hopf algebras and $K$-homology of Grassmannians. Int. Math. Res. Not. IMRN, (24):Art. ID rnm125, 48, 2007. ISSN 1073-7928. doi: 10.1093/imrn/rnm125. URL http://dx.doi.org/10.1093/imrn/rnm125.

A. Lascoux and M.-P. Schützenberger. Le monoïde plaxique. In Noncommutative structures in algebra and geometric combinatorics (Naples, 1978), volume 109 of Quad. "Ricerca Sci.", pages 129-156. CNR, Rome, 1981.

A. Lascoux and M.-P. Schützenberger. Symmetry and flag manifolds. In Invariant theory (Montecatini, 1982), volume 996 of Lecture Notes in Math., pages 118-144. Springer, Berlin, 1983. doi: 10.1007/ BFb0063238. URL http://dx.doi.org/10.1007/BFb0063238.

C. Lenart. Combinatorial aspects of the $K$-theory of Grassmannians. Ann. Comb., 4(1):67-82, 2000. ISSN 0218-0006. doi: 10.1007/PL00001276. URL/http://dx.doi.org/10.1007/PL00001276

O. Pechenik and A. Yong. Equivariant K-theory of Grassmannians. ArXiv e-prints, June 2015.

J. B. Remmel and M. Shimozono. A simple proof of the Littlewood-Richardson rule and applications. Discrete Math., 193(1-3):257-266, 1998. ISSN 0012-365X. doi: 10.1016/S0012-365X(98)00145-9. URL http://dx.doi.org/10.1016/S0012-365X(98)00145-9. Selected papers in honor of Adriano Garsia (Taormina, 1994).

G. d. B. Robinson. On the Representations of the Symmetric Group. Amer. J. Math., 60(3):745-760, 1938. ISSN 0002-9327. doi: 10.2307/2371609. URL http://dx.doi.org/10.2307/2371609

C. Schensted. Longest increasing and decreasing subsequences. Canad. J. Math., 13:179-191, 1961. ISSN 0008-414X.

M.-P. Schützenberger. La correspondance de Robinson. In Combinatoire et représentation du groupe symétrique (Actes Table Ronde CNRS, Univ. Louis-Pasteur Strasbourg, Strasbourg, 1976), pages 59113. Lecture Notes in Math., Vol. 579. Springer, Berlin, 1977.

R. P. Stanley. Enumerative combinatorics. Vol. 2, volume 62 of Cambridge Studies in Advanced Mathematics. Cambridge University Press, Cambridge, 1999. ISBN 0-521-56069-1; 0-521-78987-7. doi: 10.1017/CBO9780511609589. URL http://dx.doi.org/10.1017/CB09780511609589. With a foreword by Gian-Carlo Rota and appendix 1 by Sergey Fomin.

J. R. Stembridge. A concise proof of the Littlewood-Richardson rule. Electron. J. Combin., 9(1):Note 5, 4 pp. (electronic), 2002. ISSN 1077-8926. URL/http://www. combinatorics.org/Volume_ 9/Abstracts/v9iln5.html 